\title{
NOTE
}

\section{On the Intersection of a Set of Direction Cones}

\author{
KAI TANG \\ University of Michigan, EECS Department, Ann Arbor, Michigan 48109
}

Received April 4, 1988; accepted May 31, 1988

\begin{abstract}
A new method is suggested to compute the intersection of a set of direction cones encountered in the problem of passing a convex polyhedron through a window. The time requirement of this method is $O(\mathrm{~nm})$, where $n$ is the number of vertices of the polyhedron and $m$ is the number of vertices of the window. Besides this time improvement, the concept of parallel congruence, which the new method is crucially based on, is discussed. 1989 Academic Press, Inc.
\end{abstract}

\section{INTRODUCTION}

The problem of passing a convex polyhedron through a window is described as: Given a convex polyhedron $P=\left(p_{1}, p_{2}, \ldots, p_{n}\right)$ and a convex polygon $W=$ $\left(w_{1}, w_{2}, \ldots, w_{m}\right)$ on a plane $h$ not intersecting $P$, find all the directions for translating (single translation) $P$ through $W$.

Toussaint [1] proposed an $O(n m \log n m)$ algorithm to solve this problem. He first showed that $P$ can pass $W$ with a single translation in direction $\theta$ if, and only if, each vertex of $P$ can be passed through $W$ with a single translation in direction $\theta$. Based on this observation, he posed the algorithm as follows: Consider vertex $p_{i}$ of $P$. All directions for translating $p_{i}$ from its initial position through window $W$ are defined by all the vectors emanating at $p_{i}$ and intersecting $H$ in $W$. Therefore the cone determined by the half-lines from $p_{i}$ through $w_{j}, j=1,2, \ldots, m$ specifies all such directions for $p_{i}$. This cone is named as direction cone of $p_{i}$ and is denoted as $\operatorname{CONE}\left(p_{i}, W\right)$. Construct a 3D euclidean direction space $D$ and translate all the cones $\operatorname{CONE}\left(p_{i}, W\right), i=1,2, \ldots, n$ in $D$ such that the $p_{i}$ all overlap with the origin of $D$. Then the intersection of all the cones in $D$ gives another cone which is the set of directions for simultaneous translation of all the $p_{i}$, and hence of $P$. Each cone can be computed in $O(\mathrm{~m})$ time and thus all the cones can be found in $O(\mathrm{~nm})$ time. All the cones can be translated to $D$ in $O(\mathrm{~nm})$ time. To compute the intersection of the cones, Toussaint views each cone as the intersection of $m$ half spaces determined by the planes coplanar with the $m$ faces of each cone. The interior half space contains the cone. Therefore the solution cone is the intersection of all the interior half spaces determined by all the cones in $D$. Since the intersection of $k$ half spaces in 3D space can be computed in $O(k \log k)$ time (Preparata and Muller [3]), the overall time requirement is $O(m n \log m n)$.

The major computation in the algorithm cited above is to find the intersection of cones. As long as it is viewed as an intersection of $m n$ half spaces, the time requirement cannot be improved since $O(k \log k)$ is the optimal to compute the intersection of $k$ half spaces.

Finding the intersection of a set of half spaces is a general scheme allowing half spaces having arbitrary positions and orientations. In our case, however, those $\mathrm{nm}$ 


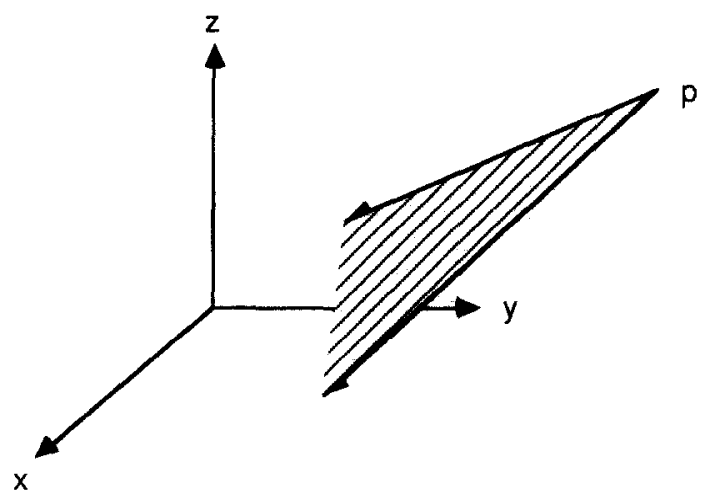

FIG. 1. An open triangle.

half spaces all overlap with a single point (the origin of $D$ ). With this constraint, it is natural to ask if a faster computational approach, rather than simply intersecting half spaces, to finding the solution cone is possible. The author's answer is yes. As we will see, by utilizing a concept called parallel congruence $O(\mathrm{~nm})$ time is enough to compute the intersection of those $n$ cones and hence the whole problem can be solved in $O(\mathrm{~nm})$ time.

\section{PRELIMINARIES}

First let us define a geometric item called open triangle (or simply OT) as: an open triangle OT is an infinite planar region bounded by two rays emanating from a point $p$, called the origin of that OT, in 3D space. Figure 1 shows an example.

Each direction cone is an open prism bounded by $m$ such OTs. The intersection of those OTs of a cone with plane $H$ constitutes convex $m$-gon $W$. If a direction cone is translated to a new position, the intersection between $H$ and the cone's $m$ translated OTs is still a convex $m$-gon. The following theorem tells the relation between the two polygons. For the sake of discussion, suppose a convex $k$-gon is represented as $\left(e_{1}, e_{2}, \ldots, e_{k}\right)$, where each $e_{i}$ is an edge of the polygon and a clockwisely sequential succeeding of $e_{1}, e_{2}, \ldots, e_{k}$ constitutes the polygon. The direction of an edge $e_{i}$ is decided such that the polygon lies to its right.

THEOREM 1. Given a direction cone bounded by m OTs, with the OTs' origin at a point $p$ and their intersection with a plane $H$ being a convex m-gon $W=$ $\left(w_{1}, w_{2}, \ldots, w_{m}\right)$. If this cone is translated to a new position with the new origin at $p^{\prime}$, the intersection between $H$ and $m$ translated OTs is still a convex $m-g o n W^{\prime}=$ $\left(w_{1}^{\prime}, w_{2}^{\prime}, \ldots, w_{m}^{\prime}\right)$, and each edge $w_{i}^{\prime}, i=1,2, \ldots, m$ is parallel to $w_{i}$ and has the same direction as $w_{i}$.

Proof. The convexity and having $m$ edges of $W^{\prime}$ is conceivable. Consider an open triangle $\mathrm{OT}_{i}$. Its intersection with $H$ is edge $w_{i}$. The derivation of this edge can be thought of two steps: first the plane coplanar with $\mathrm{OT}_{i}$ intersects $H$ and generates an infinite line $l$; then the two rays emanating from point $p$ intersect $l$ and delimits $e_{i}$. Suppose the origin of $\mathrm{OT}_{i}$ is moved to $p^{\prime}$ and $\mathrm{OT}_{i}$ becomes $\mathrm{OT}_{i}^{\prime}$, as shown in Fig. 2. Since the cone is under pure translation, the normal of the plane of 


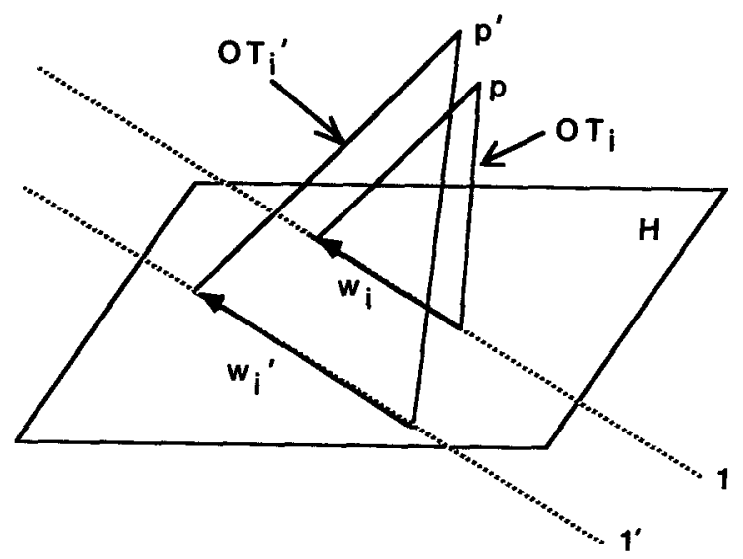

FIG. 2. Parallelism between edges $w i$ and $w i^{\prime}$.

$\mathrm{OT}_{i}^{\prime}$ should be the same as that of $\mathrm{OT}_{i}$ and correspondingly the intersecting line $l^{\prime}$ of $\mathrm{OT}_{i}^{\prime}$ plane with $H$ must be parallel to $l$, only the length of edge $w_{i}^{\prime}$ may be different to that of $w_{i}$. The equality of the directions is obvious.

Polygons $W$ and $W^{\prime}$ actually bear an important characterization; parallel congruence, as we defined below.

Definition. Two convex polygons $P=\left(p_{1}, p_{2}, \ldots, p_{m}\right)$ and $Q=$ $\left(q_{1}, q_{2}, \ldots, q_{k}\right), m \leq k$, are said parallel congruent to each other if and only if for each edge $p_{i}$ there is an edge $q_{j}$ such that $p_{i}$ and $q_{j}$ are parallel and have the same direction.

Figure 3 demonstrates two examples of parallel congruent convex polygons.

If two convex polygons are parallel congruent to each other, it is interesting to ask what would their intersection look like. Generally, the intersection of an arbitrary convex $l$-gon and an arbitrary $m$-gon is a convex polygon having up to $1+m$ vertices.

THEOREM 2. If a convex l-gon $P$ and a convex m-gon $Q$ are parallel congruent to each other, their intersection is a convex polygon with at mast $\operatorname{Max}\{l, m\}$ vertices and it is parallel congruent to $P$ and $Q$ too.

Proof. Without loss of generality, assume $l=m . P=\left(p_{1}, p_{2}, \ldots, p_{m}\right), Q=$ $\left(q_{1}, q_{2}, \ldots, q_{m}\right)$. Let the intersection of $P$ and $Q$ be $C=\left(c_{1}, c_{2}, \ldots, c_{r}\right)$. First we note since each $c_{i}$ is either an edge of $P$ (or $Q$ ) or a part of an edge of $P$ (or $Q$ ), the
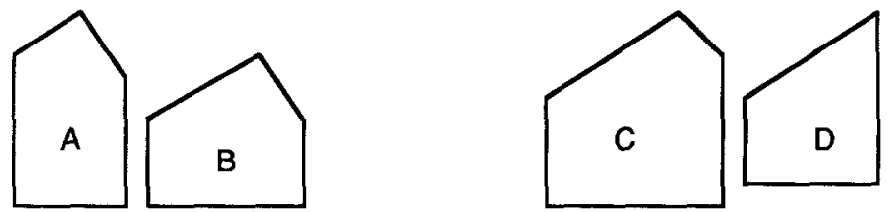

Fig. 3. $A$ and $B, C$ and $D$ are parallel congruent to each other respectively. 


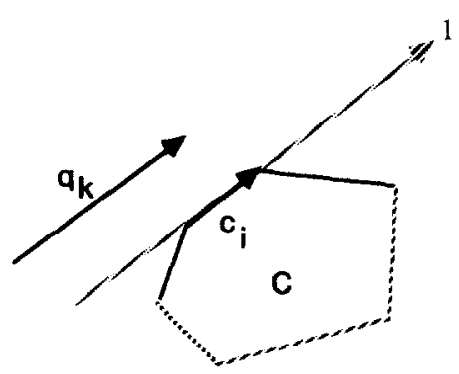

(a)

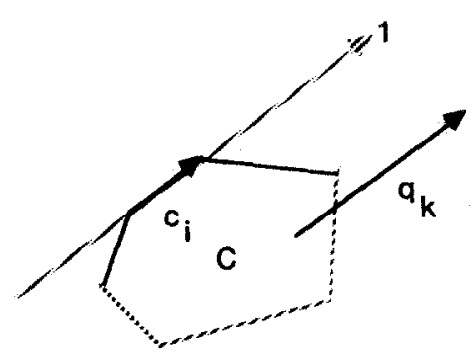

(b)

Fig. 4. Mutual exclusion property.

direction of $c_{i}$ must be the same as that of the constituting edge of $P$ (or $Q$ ). Second, due to the convexity each edge of $P$ and $Q$ can constitute at most one edge of $C$. Now consider Fig. 4. Suppose $c_{i}$ is constituted by edge $p_{j}$ and edge $q_{k}$ is parallel congruent to $p_{j}$. The extension ray line $l$ of $c_{i}$ partitions the plane into two half planes. If $q_{k}$ is to the left of $l$, as in (a), it will never intersect any edges of $P$ due to convexity, neither can it be an edge of $C$. Conversely, if $q_{k}$ is to the right of $l$, as in (b), either $P$ and $Q$ do not intersect at all, or the whole polygon $C$ is to the right of $q_{k}$ because of convexity. In both cases $c_{i}$ and hence $p_{j}$ cannot be an edge of $C$, contradicting our assumption. (In the degenerate case when $q_{k}$ colides with $l$, at most one edge of $C$ can be constituted by $P$ and $Q$.) This mutual exclusion between $p_{j}$ and $q_{k}$ implies that each pair of parallel congruent edges of $P$ and $Q$ can constitute at most one edge of $C$. Therefore $r \leq \operatorname{Max}\{l, m\}$. The proof of the parallel congruence between $C$ and $P, Q$ is trivial.

Q.E.D.

\section{THE ALGORITHM}

With all the preliminaries discussed above, we are now ready to present the algorithm. The main idea is, instead of intersecting the defining half spaces of those cones, the $m$ parallel congruent polygons that are generated by the intersections between plane $H$ and the cones are intersected first and then the resultant convex polygon is mapped back to the origin of $D$. Suppose polyhedron $P$ has $n$ vertices, window $W$ has $m$ vertices and lies on plane $H$.

Procedure Find All $(P, Q, W)$.

Th find the solution cone of a polyhedron $P$ and a window $W \%$

Step 1. For each vertex $p_{i}$ of $P$ constitute its direction cone $\operatorname{CONE}\left(p_{i}, W\right)$;

Step 2. Translate all the $n$ direction cones to a particular vertex point, say $p_{1}$;

Step 3. Intersect each translated cone with plane $H$ and let array $H P[1: n]$ store those resultant convex m-gons;

Step 4. $I W \leftarrow H P[1]$

For $i=2$ to $n$ do

$I W \leftarrow I W$ intersecting $H P[i]$;

Step 5. Draw rays emanating from $p_{1}$ and going through each vertex of $I W$, constitute the solution cone from these rays,

Step 6. return the solution cone

end Find All.

Complexity Analysis. Step 1 runs $O(\mathrm{~nm})$ time. Step 2 also runs $O(\mathrm{~nm})$ time, since for each direction cone we have to translate its $m$ OTs. Step 3 is $O(n m)$. In 
step 4, $n-1$ intersections of two parallel congruent convex polygons $I W$ and $H P[i], i=2,3, \ldots, n$ are performed. The intermediate intersection polygon $I W$ has at most $m$ edges. Each of these intersection takes at most $O(2 m)=O(m)$ time (refer to Preparata and Shamos [2]), resulting in an $O(\mathrm{~nm})$ time upper bound for step 4. Step 5 runs at most $O(m)$ time. Overall the algorithm Find All takes $O(\mathrm{~nm})$ time.

\section{CONCLUSION}

In this paper, a new approach for finding the intersection of direction cones encountered in the problem of passing a convex polyhedron through a convex window is proposed. Besides the time improvement from $O(n m \log n m)$, as originally given by Toussaint [1], to $O(\mathrm{~nm})$, the concept of parallel congruence introduces a new subset of convex polygons. The mutual exclusion property among parallel congruent polygons is not only useful in finding intersection of convex polygons, but might also helpful, as the author expects, in solving some other computational geometry problems.

\section{REFERENCES}

1. G. T. Toussaint, Movable separability of sets, in Computational Geometry (G. Toussaint, Ed.), pp. 335-375, North-Holland, Amsterdam, 1985.

2. F. P. Preparata and M. Shamos, Computational Geometry, Springer-Verlag, New York/Berlin, 1985.

3. F. P. Preparata and D. E., Muller, Finding the intersection of $n$ half-spaces in time $O(n \log n)$, Theoret. Comput. Sci. 8, 1979, 45-55. 